\title{
Stability of perturbed linear distributed parameter systems
}

\section{Pandolfi *}

Politecnico di Torino, Dipartimento di Matematica, Corso Duca degli Abruzzi 24, 10129 Torino. Italy

\section{H. Zwart}

Universiteit Twente, Fakulteit der Toegepaste Wiskunde, Postbus 217, 7500 AE Enschede, Netherlands

Received 15 August 1990

Revised 15 February and 23 May 1991

Abstract: We present an estimate for a class of unbounded perturbations of the generator of an exponentially stable semigroup. When this estimate is satisfied then the perturbed operator still generates an exponentially stable semigroup. For generators of holomorhic semigroups the class of allowed perturbations contains unbounded operators of the form $H A^{\alpha}$, with $\alpha<\frac{1}{2}$. The estimate is given in terms of the norm of the solution of the Lyapunov equation which corresponds to the unperturbed system.

Keywords: Distributed parameter systems; stability; robustness: Lyapunov equation; $C_{0}$-semigroup.

\section{Introduction}

In this note $A$ represents the generator of a $C_{0}$-semigroup $E(t)$ on a Hilbert space $X$. Our first assumption is that this semigroup is exponentially stable, namely:

Assumption 1. There exist numbers $M>1, \gamma>0$ such that

$\|E(t) x\|<M \mathrm{e}^{-\gamma t}\|x\| \quad \forall x \in X$.

We shall say, for simplicity, that $A$ is a stable operator.

\footnotetext{
* Paper written with financial support of the Italian Ministero della Ricerca Scientifica e Tecnologica within the program of GNAFA-CNR.
}

Further assumptions on the operator $A$ will be presented in the next section, where we study the robustness of the stability property under a class of unbounded perturbations.

The operator $A$ represents the nominal model of some process, whose exact description is often given by an operator of the form $A+K$ and

$$
K: \operatorname{dom}(K) \subset X \rightarrow X
$$

(the 'perturbation') is only imperfectly known. As the perturbation $K$ may affect the stability properties of the system described by $A$, many efforts have been devoted to give conditions under which $A+K$ generates a $C_{0}$-semigroup $E_{K}(t)$ which still is exponentially stable. We quote in particular the paper [5], where the 'stability radius' is exactly characterized for finite-dimensional systems. It is proved in this paper that if

$$
\|K\|<\min _{\omega}\left(1 /\left\|(\mathrm{i} \omega I-A)^{-1}\right\|\right)=\mathscr{R},
$$

then $A+K$ is a stable operator; and, there exists a perturbation $K$ whose norm is $\mathscr{R}$ and such that $A+K$ is not exponentially stable (the number $\mathscr{R}$ is the unstructured stability radius of $A$ ). This result is extended to 'structured' perturbations in [6] and to distributed systems in [10,11].

A different approach had been previously proposed - for finite dimensional systems - in [8] and pursued in other papers (see for example [13]; see [1] for a recent paper). Namely, it was noted that $A+K$ is stable provided that the Lyapunov equation for $A+K$ admits a positive solution which could be used to construct a Lyapunov function for the perturbed system.

It is also true for distributed parameter systems that exponential stability of $E(t)$ is characterized by the existence of a self-adjoint positive solution $\Pi$ to the Lyapunov equation [2], which takes the form

$$
\begin{aligned}
& \langle A x, P y\rangle+\langle x, P A y\rangle=-\langle x, y\rangle \\
& \forall x, y \in \operatorname{dom}(A) .
\end{aligned}
$$


Consequently, under Assumption 1, Lyapunov equation (LE) admits a unique solution (which is bounded positive operator). It is given by

$$
\langle x, \Pi y\rangle=\int_{0}^{+\infty}\langle E(t) x, E(t) y\rangle \mathrm{d} t .
$$

So that

$$
\|\Pi\|=\sup _{\|x\|=1} \int_{0}^{+\infty}\|E(t) x\|^{2} \mathrm{~d} t=: \Lambda^{2}<\infty .
$$

Remark 1. (a) Conversely, from [2], if a non-negative solution $P=P^{*}$ exists to (LE), then the semigroup generated by $A$ is exponentially stable and the above integral representation holds.

(b) We see from the integral representation of $\Pi$ that $\operatorname{Ker} \Pi=\{0\}:$ if $\Pi x=0$ then $E(t)=0$ for almost all values of $t$. Hence, since $t \rightarrow E(t) x$ is a continuous function, $x=E(0) x=0$. We shall use this property in the proof of our main result.

The norm of the operator $\Pi$ can be used to derive a condition under which $A+K$ still is a stable operator:

Theorem 1. Let $K$ be a bounded operator. If $\|K\|$ $<1 / 2\|\Pi\|$, then $A+K$ is stable.

We shall see in the following that this result, proved in [8] for finite dimensional systems, also holds for $\operatorname{dim} X=+\infty$ and bounded operators $K$, without further assumptions on the operator $A$ (the proof that we shall present is different from the one in [8]).

The main concern of this paper is to present an extension of Theorem 1 to a class of unbounded perturbations (next section). This will require suitable assumptions on the operator $A$ since in this case the following difficulties must be overcome:

- $A+K$ may not be a generator;

- the norm of $K$ is not defined.

We observe that, however, unbounded perturbations are of the greatest importance for the applications, since they can be used to represent perturbations on the coefficients of a differential operator or on a delayed term for a time-lag system.
The class of the systems that can be treated with the techniques in this paper is a subset of that studied in $[10,11]$ as we shall see in Section 3. In spite of this, we believe that the results in this paper can be useful since, as a by-product, we get not only stability for the perturbed systems, but also an estimate for the $L^{2}$-norm of the semigroup generated by $A+K$, as discussed in Section 3 . Consequently, we also get an estimate for the norm of the solutions of the Lyapunov equation for the perturbed system. This result, which cannot be obtained by the mere knowledge of the stability radius, is interesting since the state-space approach to $H^{\infty}$-control theory heavily relies on solutions of Lyapunov (and Riccati) equations.

\section{Unbounded perturbations}

The main case that we have in mind is the case that $A$ generates a holomorphic semigroup; however, the properties that we need can be abstracted as follows:

Assumption 2. The perturbation $K$ belongs to a linear space $\mathscr{P}$ of operators, with the following properties:

P1. $\operatorname{dom}(K) \supseteq \operatorname{dom}(A)$. The operator $A+K$ is the generator of a $C_{0}$-semigroup $E_{K}(t)$ on $X$.

P2. For each $K \in \mathscr{P}$ the number $L_{K}$ defined by

$$
\begin{aligned}
L_{K}^{2}=\sup \left\{\int_{0}^{+\infty} \|\right. & K E(t) x \|^{2} \mathrm{~d} t \\
& x \in \operatorname{dom}(A),\|x\|=1\}
\end{aligned}
$$

is finite.

P3. If $K \in \mathscr{P}$, then $K$ is $A$-bounded, i.e. there exist numbers $\alpha, \beta$ such that

$$
\|K x\| \leq \alpha\|x\|+\beta\|A x\| \quad \forall x \in \operatorname{dom}(A) .
$$

Of course, if $\mathscr{P}$ is $L(X)$ the previous assumptions are satisfied without any restriction on the generator $A$ of the exponentially stable semigroup $E(t)$. In this case, $L_{K} \leq\|K\| \cdot \Lambda$. Otherwise, the operator $A$ must be in a suitable class and the previous assumptions are satisfied when $A$ generates a holomorphic semigroup, and if $\mathscr{P}$ is the class of the operators $K$ which are linear combinations of operators of the form $K=H A^{\alpha}$, with 
$0 \leq \boldsymbol{\alpha}<\frac{1}{2}$. Here $H$ is a bounded operator. In fact, $\operatorname{dom}(K) \supseteq \operatorname{dom}(A)$ and $[9$, Corollary 2.4, p. 81] $A+K$ generates a holomorphic semigroup. Moreover, for $K=H A^{\alpha}$,

$$
\begin{aligned}
\|K E(t)\| & \leq\|H\| \cdot\left\|A^{\alpha} E(t)\right\| \\
& \leq\left(\|H\| M_{\alpha}\right) \mathrm{e}^{-\gamma t} / t^{\alpha} .
\end{aligned}
$$

Here, the number $\gamma$ is the number which appears in Assumption 1 and, for $\|x\| \leq 1$,

$$
\begin{aligned}
& \int_{0}^{+\infty}\|K E(t) x\|^{2} \mathrm{~d} t \\
& \quad \leq\|H\| M_{\alpha} \int_{0}^{+\infty} \mathrm{e}^{-2 \gamma t} / t^{2 \alpha} \mathrm{d} t
\end{aligned}
$$

The integral is finite, since $2 \alpha<1$ and $\gamma$ is positive.

Theorem 6.10 in [9] implies that $K$ is $A$ bounded.

The case just described, that $A$ generates a holomorphic semigroup and $K$ of the form $H A^{\alpha}$, is not the unique case that can be treated with the techniques of this paper. A second example is the following one: $A$ is an arbitrary infinitesimal generator of an exponentially stable semigroup and $K$ is a closed operator with $\operatorname{dom}(K) \supseteq \bigcup_{t>0} E(t) X$, and

$\int_{0}^{1}\|K E(t)\| \mathrm{d} t<+\infty$.

A perturbation with these properties is a class $\mathscr{P}$ perturbation [3, p.70]. In this case $A+K$ generates a semigroup and $K$, being closed, is $A$-bounded. From (3) and the exponential stability of $E(t)$ one can easily prove $\mathrm{P} 2$ of Assumption 2.

Consequently, from now on we assume that the class $\mathscr{P}$ of the perturbations satisfies properties $\mathrm{P} 1-\mathrm{P} 3$. The main result of this paper is the following extension of Theorem 1 . We recall that $\Lambda$ is the number of defined in (2).

Theorem 2. If $K \in \mathscr{P}$ and $L_{K}<1 / 2 \Lambda$, then the operator $A+K$ generates an exponentially stable semigroup.

For the proof we need to consider the perturbed Lyapunov equation, i.e. the equation

$$
\begin{gathered}
\langle(A+K) x, P y\rangle+\langle x, P(A+K) y\rangle \\
=-\langle x, y\rangle \quad \forall x, y \in \operatorname{dom}(A) .
\end{gathered}
$$

But, we need the integral form of the perturbed Lyapunov equation. We introduce the operator

$$
\begin{aligned}
& \langle x, F(P) y\rangle \\
& -\langle x, \Pi y\rangle+\int_{0}^{+\infty}\langle K E(t) x, P E(t) y\rangle \mathrm{d} t \\
& \quad+\int_{0}^{+\infty}\langle P E(t) x, K E(t) y\rangle \mathrm{d} t .
\end{aligned}
$$

Lemma 3. If $K \in \mathscr{P}$, then the transformation $F(P)$, defined by (5) for $x, y \in \operatorname{dom}(A)$, has a bounded extension to $X$ for each $P \in L(X)$.

Proof. Clearly, for $x, y \in \operatorname{dom}(A)$ with $\|x\|=$ $\|y\|=1$ we have

$$
\begin{aligned}
& \left|\int_{0}^{+\infty}\langle K E(t) x, P E(t) y\rangle \mathrm{d} t\right| \\
& \quad \leq\left\{\int_{0}^{+\infty}\|K E(t) x\|^{2} \mathrm{~d} t\right\}^{1 / 2} \cdot\|P\| \cdot \Lambda \\
& \quad \leq L_{K} \cdot\|P\| \cdot \Lambda .
\end{aligned}
$$

Hence, the first integral in (5) has a bounded extension. Analogously it is seen that the second integral has a bounded extension.

Furthermore, we have:

Lemma 4. An operator $P$ solves the equation in (4) if and only if $P=F(P)$.

Proof. We assume first that $P$ is a solution to (4) so that for $x, y \in \operatorname{dom}(A)$ we have

$$
\begin{aligned}
\frac{\mathrm{d}}{\mathrm{d} t} & \langle E(t) x, P E(t) y\rangle \\
= & \langle A E(t) x, P E(t) y\rangle+\langle E(t) x, P A E(t) y\rangle \\
= & -\langle E(t) x, E(t) y\rangle-\langle K E(t) x, P E(t) y\rangle \\
& -\langle E(t) x, P K E(t) y\rangle .
\end{aligned}
$$

As $E(t) x$ decays exponentially for $t \rightarrow+\infty$, after integration we have

$$
\begin{aligned}
\langle x, P y\rangle= & \langle x, \Pi y\rangle \\
& +\int_{0}^{+\infty}\langle K E(t) x, P E(t) y\rangle \mathrm{d} t \\
& +\int_{0}^{+\infty}\langle P E(t) x, K E(t) y\rangle \mathrm{d} t
\end{aligned}
$$


for $x, y \in \operatorname{dom}(A)$, i.e. $P=F(P)$ since $\operatorname{dom}(A)$ is dense in $X$ and $P, F(P)$ are bounded operators.

For the converse implication we use the $A$ boundedness of $K$. Let us assume that $P$ is a fixed point of $F(\cdot), P=F(P)$. Then, for $x, y \in$ $\operatorname{dom}\left(A^{2}\right)$,

$$
\begin{aligned}
\langle A x, & P y\rangle+\langle x, P A y\rangle \\
= & \langle A x, \Pi y\rangle+\int_{0}^{+\infty}\langle K E(t) A x, P E(t) y\rangle \mathrm{d} t \\
& +\int_{0}^{+\infty}\langle P E(t) A x, K E(t) y\rangle \mathrm{d} t \\
& +\langle x, \Pi A y\rangle \\
& +\int_{0}^{+\infty}\langle K E(t) x, P E(t) A y\rangle \mathrm{d} t \\
& +\int_{0}^{+\infty}\langle P E(t) x, K E(t) A y\rangle \mathrm{d} t .
\end{aligned}
$$

We note that these expressions make sense since if $x \in \operatorname{dom}\left(A^{2}\right)$ then

$E(t) A x \in \operatorname{dom}(A) \subseteq \operatorname{dom}(K)$.

Moreover, from (LE),

$\langle A x, \Pi y\rangle+\langle x, \Pi A y\rangle=-\langle x, y\rangle$.

Let us consider the sum of the first and the third integral. Formally it is

$$
\int_{0}^{+\infty} \frac{\mathrm{d}}{\mathrm{d} t}\langle K E(t) x, P E(t) y\rangle \mathrm{d} t=-\langle K x, P y\rangle .
$$

Analogously, the remaining two integrals are equal to $-\langle P x, K y\rangle$. We prove now that these formal equalities indeed hold in two steps:

(a) We prove that

$$
\begin{aligned}
\frac{\mathrm{d}}{\mathrm{d} t}\langle & K E(t) x, P E(t) y\rangle \\
= & \langle K E(t) A x, P E(t) y\rangle \\
& +\langle K E(t) x, P E(t) A y\rangle .
\end{aligned}
$$

We recall that $x, y$ belong to $\operatorname{dom}\left(A^{2}\right)$ and we note

$$
\frac{\mathrm{d}}{\mathrm{d} t} K E(t) x=K E(t) A x .
$$

This is true since, with $y=E(t) x$,

$$
\begin{aligned}
& \left\|\frac{K[E(t+h) x-E(t) x]}{h}-K E(t) A x\right\| \\
& =\left\|K\left\{\frac{[E(h) y-y]}{h}-A y\right\}\right\| \\
& \leq \alpha\left\|\frac{E(h) y-y}{h}-A y\right\| \\
& \quad+\beta\left\|\frac{E(h) z-z}{h}-A z\right\|,
\end{aligned}
$$

where $z=A y \in \operatorname{dom}(A)$ since $x \in \operatorname{dom}\left(A^{2}\right)$. The limits of both the last sums are zeros for $h \rightarrow 0$.

(b) Consequently,

$$
\begin{aligned}
& \int_{0}^{T} \frac{\mathrm{d}}{\mathrm{d} t}\langle K E(t) x, P E(t) y\rangle \mathrm{d} t \\
& \quad=\langle K E(T) x, P E(t) y\rangle-\langle K x, P y\rangle
\end{aligned}
$$

and

$$
\begin{aligned}
&|K E(T) x, P E(T) y\rangle \mid \\
& \leq\|K E(T) x\| \cdot\|P E(T) y\| \\
& \leq\{\alpha\|E(T) x\|+\beta\|E(T) A x\|\} \\
& \cdot\|P\| \cdot\|E(T) y\| \\
& \rightarrow 0 \text { for } T \rightarrow+\infty .
\end{aligned}
$$

This shows that $P$ satisfies (4), with $x, y$ in $\operatorname{dom}\left(A^{2}\right)$, a dense subset of the domain of $A$.

In fact, equation (4) also holds with $x, y$ in $\operatorname{dom}(A)$, as we shall see now. We note that (4) shows that

$$
\langle(A+K) x, P y\rangle=-\langle x,[P(A+K) y+y]\rangle
$$

Let $y_{0}$ be fixed in $\operatorname{dom}\left(A^{2}\right)$ and $\left\{x_{n}\right\} \in \operatorname{dom}\left(A^{2}\right)$ be a sequence which converges to $x_{0} \in \operatorname{dom}(A)$. Then,

$$
\begin{aligned}
& \left\langle(A+K) x_{0}, P y_{0}\right\rangle-\left\langle(A+K) x_{n}, P y_{0}\right\rangle \\
& \quad=-\left\langle x_{0}-x_{n},\left[P(A+K) y_{0}+y_{0}\right]\right\rangle \rightarrow 0 .
\end{aligned}
$$

This implies that (4) is satisfied with $x=x_{0}$ and $y_{0}$. Now fix $x_{0}$ in $\operatorname{dom}(A)$ and repeat the previous construction with a sequence $\left\{y_{n}\right\}$ from $\operatorname{dom}\left(A^{2}\right)$, which converges to an element $y$ of $\operatorname{dom}(A)$.

A first step in the proof of Theorem 2 requires that $F(\cdot)$ must have a fixed point $P$ (it is easy to see that if $P$ is a fixed point also $P^{*}$ is a fixed 
point so that uniqueness implies that $P$ is self-adjoint).

Lemma 5. If $L_{K}<1 / 2 \Lambda$, then $F(\cdot)$ is a contraction. In this case, its fixed point $P_{K}$ satisfies the following estimates:

(a) $\left\|P_{K}\right\| \leq\|\Pi\| /\left(1-2 L_{K} \Lambda\right)$.

(b) If also $L_{K^{\prime}}<1 / 2 \Lambda$, there exists a number $\alpha=\alpha\left(K, K^{\prime}\right)$ such that

$\left\|P_{K}-P_{K^{\prime}}\right\| \leq \alpha L_{\left(K-K^{\prime}\right)}$.

The number $\alpha$ is given by (12) below.

Proof. Clearly,

$$
\begin{aligned}
& \langle x, F(P) y\rangle \cdot\langle x, F(Q) y\rangle \\
& =\int_{0}^{+\infty}\langle K E(t) x,[P-Q] E(t) y\rangle \mathrm{d} t \\
& \quad+\int_{0}^{+\infty}\langle[P-Q] E(t) x, K E(t) y\rangle \mathrm{d} t .
\end{aligned}
$$

Let us assume that $\|x\|$ and $\|y\|$ are less than or equal to 1 . The Schwarz inequality applied to both the integrals shows that each one of them is less than $L_{K} \Lambda \cdot\|P-Q\|$ so that we have a contraction if $2 L_{K} \Lambda<1$.

Let $Q$ be zero and $P_{K}=F\left(P_{K}\right)$. Then, as $F(0)$ $=\Pi$, the previous inequality shows that

$$
\left\|P_{K}\right\|=\left\|F\left(P_{K}\right)\right\| \leq\|\Pi\|+2 L_{K} \Lambda \cdot\left\|P_{K}\right\|,
$$

i.e. $\left\|P_{K}\right\| \leq\|\Pi\| /\left(1-2 L_{K} \Lambda\right)$ when the denominator is positive.

Let us consider now two operators $K, K^{\prime}$ from the class $\mathscr{P}$, such that both $L_{K}$ and $L_{K^{\prime}}$ are less than $1 / 2 \Lambda$. Let $P, P^{\prime}$ be the solutions of the corresponding perturbed Lyapunov equations, so that

$$
\begin{aligned}
& \left\langle x .\left(P-P^{\prime}\right) y\right\rangle \\
& =\int_{0}^{+\infty}\langle K E(t) x, P E(t) y\rangle \mathrm{d} t \\
& \quad+\int_{0}^{+\infty}\langle P E(t) x, K E(t) y\rangle \mathrm{d} t \\
& \quad-\int_{0}^{+\infty}\left\langle K^{\prime} E(t) x, P^{\prime} E(t) y\right\rangle \mathrm{d} t \\
& \quad-\int_{0}^{+\infty}\left\langle P^{\prime} E(t) x, K^{\prime} E(t) y\right\rangle \mathrm{d} t .
\end{aligned}
$$

Let is zonsider the difference of the first and the third integral. This can be written in the following form:

$$
\begin{aligned}
& \int_{0}^{+\infty}\left\langle K E(t) x,\left(P-P^{\prime}\right) E(t) y\right\rangle \mathrm{d} t \\
& \quad+\int_{0}^{+\infty}\left\langle\left(K-K^{\prime}\right) E(t) x, P^{\prime} E(t) y\right\rangle \mathrm{d} t .
\end{aligned}
$$

The modulus of this number is less than

$$
\begin{aligned}
& L_{K} \Lambda \cdot\left\|P-P^{\prime}\right\| \cdot\|x\| \cdot\|y\| \\
& \quad+\left\{\int_{0}^{+\infty}\left\|\left(K-K^{\prime}\right) E(t) x\right\|^{2} \mathrm{~d} t\right\}^{1 / 2}\left\|P^{\prime}\right\| \\
& \quad \cdot \Lambda \cdot\|y\| .
\end{aligned}
$$

Hence, it is also less than

$L_{K} \Lambda \cdot\left\|P-P^{\prime}\right\|+L_{K-K^{\prime}} \cdot \Lambda \cdot \frac{\|\Pi\|}{1-2 L_{K^{\prime}} \Lambda}$

(when $\|x\|,\|y\|$ are not larger than 1). An analogous estimate holds for the difference of the remaining integrals. Consequently, the required inequality holds and the number $\alpha$, which depends both on $K$ and $K^{\prime}$, is

$\alpha=\frac{2\|\Pi\| \cdot \Lambda}{\left[1-2 L_{K} \Lambda\right]\left[1-2 L_{K}, \Lambda\right]}$.

This number is positive, since both $L_{K}$ and $L_{K}$, are less than $1 / 2 \Lambda$. We recall that $\|\Pi\|=\Lambda^{2}$.

Now that there exists a self-adjoint fixed point $P$ of the transformation $F(\cdot)$ (i.e. a self-adjoint solution of the perturbed Lyapunov equation) we only have to prove that $P$ is positive to conclude Theorem 2.

Proof of Theorem 2. Let $K_{1}$ satisfy $L_{K_{1}}<1 / 2 \Lambda$ and consider the operators $K_{\nu}:=\nu K_{1}$, for $\nu \in[0,1]$. Then $L_{K_{p}}=\nu L_{K_{1}}$, and so $K_{\nu}$ satisfies the condition $L_{K_{v}}<1 / 2 \Lambda$ too. Let $P_{\nu}$ denote the solution of (4) for $K=K_{v}$. Consider now the set

$\Xi:=\left\{\nu \in[0,1] \mid P_{\nu}>0\right\}$.

The proof is divided in two parts, in part one we shall prove that the set $\Xi$ is closed, and in part two we shall show that it is open. If we accept these results, then the proof is easy. The set $\Xi$ is an open and closed subset of $[0,1]$. That means that it is either empty or the whole space. It can 
not be empty, since 0 is in it, so it must be [0.1]. Thus $P_{1}>0$, and so $A+K_{1}$ is exponentially stable. Now we shall prove the closedness of the set $\Xi$.

The set $\Xi$ is closed. We consider the function $\nu \rightarrow P_{\nu}$. This function is continuous, since by Lemma 5 and equation (12) we have that

$$
\begin{aligned}
\left\|P_{\nu}-P_{\nu^{\prime}}\right\| & \leq \alpha L_{\left(K_{\nu}-K_{\nu^{\prime}}\right)} \\
& \leq \frac{2\|\Pi\| \Lambda}{\left[1-2 L_{K_{1}} \Lambda\right]^{2}} L_{\left(\nu-\nu^{\prime}\right) K_{1}} \\
& =\frac{2\|\pi\| \Lambda}{\left[1-2 L_{K_{1}} \Lambda\right]^{2}}\left|\nu-\nu^{\prime}\right| L_{K_{1}} .
\end{aligned}
$$

Let $\left\{\nu_{n}\right\}$ be a sequence in $\Xi$ which converges to $\nu_{0}$. Then by the above $\left\{P_{v_{n}}\right\}$ converges to $P_{\nu_{0}}$. So $P_{\nu_{0}}$ is non-negative, and since it is a solution of the Lyapunov equation it is positive (see Remark 1). So $\nu_{0}$ is an element of $\Xi$, which proves the closedness of $\Xi$.

The set $\Xi$ is open. There is one-to-one relation between $A+\nu K_{1}$ being a stable operator, and $P_{\nu}$ being positive. So we have that if $\Xi$ is not open, then there exists a $\nu_{0} \in \Xi$ such that $A+\nu_{n} K_{1}$ is not a stable operator for some sequence $\left\{\nu_{n}\right\}$ converging to $\nu_{0}$.

It is known by [12] that $A+\nu K_{1}$ is unstable if and only if $\left(s I-A-\nu K_{1}\right)^{-1}$ is not an element of $H^{\infty}(Z)$. Hence $A+\nu K_{1}$ is unstable only if there exist sequences $\left\{s_{n}^{\nu}\right\}, \operatorname{Re}\left(s_{n}^{\nu}\right) \geq 0,\left\{z_{n}^{\nu}\right\},\left\|z_{n}^{\nu}\right\|=1$ with

$\left\|\left(s_{n}^{\nu} I-A-\nu K_{1}\right) z_{n}^{\nu}\right\|$

unbounded. Defining

$x_{n}^{\nu}=\frac{\left(s_{n}^{\nu} I-A-\nu K_{1}\right)^{-1} z_{n}^{\nu}}{\left\|\left(s_{n}^{\nu} I \quad A \quad \nu K_{1}\right) z_{n}^{\nu}\right\|}$

gives a sequence $\left\{x_{n}^{\nu}\right\} \subset \operatorname{dom}(A)$ with $\left\|x_{n}^{\nu}\right\|=1$ and $\left(s_{n} I-A-\nu K_{1}\right) x_{n}^{\nu}$ converges to 0 .

With the previous result we have that there exist sequences $\left\{\nu_{n}\right\},\left\{s_{n}\right\}, \operatorname{Re}\left(s_{n}\right)>0$ and $\left\{x_{n}\right\}$ $\subset \operatorname{dom}(A),\left\|x_{n}\right\|=1$, such that $\nu_{n} \rightarrow \nu_{0}$ and $\left\{\left(s_{n} I\right.\right.$

$\left.\left.-A-\nu_{n} K_{1}\right) x_{n}\right\}$ converges to zero.

Let $P_{n}$ denote $P_{v_{n}^{\prime}}$. Then we have that

$$
\begin{aligned}
\left\langle\left(s_{n} I-A-\nu K_{1}\right) x_{n}, P_{n} x_{n}\right\rangle & \\
& +\left\langle P_{n} x_{n},\left(s_{n}-A-v_{n} K_{1}\right) x_{n}\right\rangle \\
= & 2 \operatorname{Re}\left(s_{n}\right)\left\langle x_{n}, P_{n} x_{n}\right\rangle+\left\|x_{n}\right\|^{2} .
\end{aligned}
$$

The left-hand side of this equality converges to zero (by assumption and the fact that $\left\{P_{n}\right\} \rightarrow P_{v_{0}}$, (see (13)). If $\left\{\operatorname{Re}\left(s_{n}\right)\right\}$ is bounded we can even assume $\operatorname{Re}\left(s_{n}\right) \rightarrow r_{0}$ so that the right-hand side gives

$$
\begin{aligned}
2 \operatorname{Re} & \left(s_{n}\right)\left\langle x_{n}, P_{n} x_{n}\right\rangle+\left\|x_{n}\right\|^{2} \\
= & \left(2 \operatorname{Re}\left(s_{n}\right)-2 r_{0}\right)\left\langle x_{n}, P_{n} x_{n}\right\rangle \\
& +2 r_{0}\left\langle x_{n},\left(P_{n}-P_{v_{1}}\right) x_{n}\right\rangle+2 r_{0}\left\langle x_{n}, P_{\nu_{0}} x_{n}\right\rangle \\
& +\left\|x_{n}\right\|^{2} \\
> & \frac{1}{2} \text { for } n \text { sufficiently large, }
\end{aligned}
$$

since the first two terms converge to zero, $P_{v_{0}}$ is positive and $\left\|x_{n}\right\|=1$. So, $\left\{\operatorname{Re}\left(s_{n}\right)\right\}$ must be unbounded.

We prove that this leads to a contradiction.

We know that for every $x \in \operatorname{dom}(A), K_{1} E(t) x$ is an $L_{2}$ function with norm less than or equal to $L_{K_{1}}\|x\|$. By the Paley-Wiener Theorem this implies that $K_{1}(s I-A)^{-1} x$ is in $H_{2}$ with norm less than or equal to $L_{K_{1}}\|x\|$. By Theorem 6.4.2 of [4] there holds that

$$
\left\|K_{1}(s I-A)^{-1} x\right\|^{2} \leq L_{K_{1}}^{2}\|x\|^{2} / \operatorname{Re}(s)
$$

for all $x \in \operatorname{dom}(A), \operatorname{Re}(s)>0$. Thus

$$
\left\|K_{1}\left(\begin{array}{ll}
s I & A
\end{array}\right)^{-1}\right\|^{2} \leq L_{K_{1}}^{2} / \operatorname{Re}(s) \text {. }
$$

Let $x_{n}$ denote $\left(s_{n} I-A-\nu_{n} K_{1}\right) x_{n}$. Then $\left(s_{n} I-A\right)^{-1} \nu_{n} K_{1} x_{n}=x_{n}-\left(s_{n} I-A\right)^{-1} z_{n}$

so that

$$
\begin{aligned}
& \nu_{n} K_{1}\left(s_{n} I-A\right)^{-1} \nu_{n} K_{1} x_{n} \\
& \quad=\nu_{n} K_{1} x_{n}-\nu_{n} K_{1}\left(s_{n} I-A\right)^{-1} z_{n}
\end{aligned}
$$

and hence

$$
\begin{gathered}
\left(\nu_{n} K_{1}\left(s_{n} I-A\right)^{-1}-I\right) \nu_{n} K_{1} x_{n} \\
=-v_{n} K_{1}\left(s_{n} I-A\right)^{-1} z_{n} .
\end{gathered}
$$

If $\operatorname{Re}\left(s_{n}\right) \rightarrow \infty$, then we conclude from the above that $\nu_{n} K_{1} x_{n}$ converges to zero. This with the (LE) implies that

$$
\begin{gathered}
\left\langle z_{n}+\nu_{n} K_{1} x_{n}, \Pi x_{n}\right\rangle+\left\langle\Pi x_{n}, z_{n}+\nu_{n} K_{1} x_{n}\right\rangle \\
=2 \operatorname{Re}\left(s_{n}\right)\left\langle x_{n}, \Pi x_{n}\right\rangle+\left\|x_{n}\right\|^{2},
\end{gathered}
$$


where $\Pi$ is the solution of the Lyapunov equation for $A .^{\prime}$ But the left-hand side of the above equation converges to zero, and the right-hand side is always larger than or equal to 1 , since $\left\|x_{n}\right\|=1$ and $\Pi>0$. This is a contradiction, so $\left\{\operatorname{Re}\left(s_{n}\right)\right\}$ cannot be unbounded.

\section{Concluding observations}

In the previous section we gave a condition in order that $A+K$ generates an exponentially stable semigroup which, in particular, extends the condition in [8] to distributed systems and to a class of unbounded perturbations $K$ under suitable assumptions. (The case of bounded perturbations, i.e. Theorem 1, is included as a special case. In this case $L_{K} \leq\|K\| \Lambda$.) This condition requires the knowledge of the norm of the solution $\Pi$ of a Lyapunov equation for the nominal system, which is apart from some simple examples (heated bar) not easy to solve in general. However, we quote a case in which the norm of $\Pi$ may be, at least approximately, known: it is the case when the operator $A$ has the form $A_{0}+B F$; i.e. the operator $A$ is obtained by the stabilization of an (unstable) operator $A_{0}$ and the stabilizing feedback is constructed from the solution of the regulator problem. In this case the Lyapunov equation for $A$ is the Riccati equation for the pair $(A, B)$ which is solved in order to derive the stabilizing feedback $F$.

The most important example to which our results apply is when $A$ generates a holomorphic semigroup and $K$ is a linear combination of operators of the form $H A^{\alpha}$ ( $H$ is a bounded operator and $\alpha<\frac{1}{2}$ ). It is simple to see that this class of perturbations may also be handled with the techniques in $[10,11] .\left(X=\operatorname{dom}\left(A^{\alpha}\right)=Y\right.$ and $\bar{X}=X$; the operators $B, C$ are the identity operators and $D=K$. It is easy to see that the conditions required in $[10,11]$ are satisfied.)

The results in $[10,11]$ show that the perturbed operator $A+K$ is stable if

$$
\begin{aligned}
\|K\|_{\mathscr{L}(\underline{X}, X)}<\mathscr{R}:=\min _{\omega} 1 /\left\|(\mathrm{i} \omega-A)^{-1}\right\| \\
=1 /\left\|(\mathrm{i} \omega-A)^{-1}\right\|_{\infty}
\end{aligned}
$$

and this is the best possible estimate in the sense that a destabilizing perturbation exists, whose norm is $\mathscr{R}$. If the perturbation $K$ is bounded we have from Theorem 1 stability if $\|K\|<1 / 2 \Lambda^{2}$ $=1 / 2\|\Pi\|$. This implies that $\|\Pi\|$ is not less than $1 / 2 \mathscr{R}$ :

$\|\Pi\| \geq \frac{\left\|(\mathrm{i} \omega-A)^{-1}\right\|_{\infty}}{2}$.

In the light of the importance that the solutions of Lyapunov (and Riccati) equations have in $H^{\infty}$-control theory, estimates on the norms of the solutions of the perturbed Lyapunov equations are surely important.

The Lyapunov equation approach produces further information which cannot be derived from the mere knowledge of the stability radius. It is known that exponential and $L^{2}$-stability are equivalent properties; i.e., if the operator $A$ is stable then the the solutions $x(t)=E(t) x_{0}$ of the equation $\dot{x}=A x$ are square integrable functions and $\|E(t) x\|_{L^{2}} \leq \Lambda \cdot\|x\|$. If we require that $A$ $+K$ is a stable operator then it generates a perturbed semigroup $E_{K}(t)$ such that

$\left\|E_{K^{\prime}}(t) x\right\|_{L^{2}} \leq\left\|P_{K}\right\|^{1 / 2} \cdot\|x\|$

and it is a natural condition to require that the $L^{2}$-norm of $E_{K}(t) x$ be less than a prescribed number $\Gamma$ when $\|x\| \leq 1$. It must be $\Gamma^{2}>\|\Pi\|$ since the $L^{2}$-norm is $\|\Pi\|^{1 / 2}$ for the nominal model. By Lemma 5 this condition is satisfied when

$L_{K}<\frac{\Gamma^{2}-\|\Pi\|}{2 \Lambda \Gamma^{2}}$.

\section{References}

[1] D.S. Bernstein and W.M. Haddad, Robust stability and performance analysis for state-space systems with quadratic Lyapunov bounds, SIAM J. Matrix Anal. Appl. 11 (1990) 239-271.

[2] R. Datko, Extending a theorem of Lyapunov to Hilbert spaces, J. Math. Anal. Appl. 32 (1970) 610-616.

[3] E.B. Davies, One-Parameter Semigroups (Academic Press, London, 1980).

[4] E. Hille and R.S. Phillips, Functional Analysis and Semigroups (American Mathematical Society, Providence, RI, 1957).

[5] D. Hinrichsen and A.J. Pritchard, Stability radii of linear systems, Systems Control Lett. 7 (1986) 1-10.

[6] D. Hinrichsen and A.J. Pritchard, Stability radius for structured perturbations and the algebraic Riccati equation. Systems Control Lett. 8 (1986) 105-113. 
[7] L. Pandolfi, Stability of perturbed linear distributed parameter systems: A Lyapunov equation approach, in: A. El Jai, M. Amouroux, Eds., Control and Distributed Parameter Systems (Université de Perpignan, 1989).

[8] R.V. Patel and M. Toda, Quantitative measures of robustness for multivariable systems, Joint American Control Conference, TP8-A (1980).

[9] A. Pazy, Semigroups of Linear Operators and Partial Differential Equations (Springer-Verlag, New York, 1983).

[10] A.J. Pritchard and S. Townley, A stability radius for infinite dimensional systems, in: F. Kappel, K. Kunish,
W. Schappacher, Eds, Distributed Parameter Systems, Lecture Notes in Control and Information Science No. 102 (Springer-Verlag, Berlin, 1987).

[11] A.J. Pritchard and S. Townley, Robustness of linear systems, J. Differential Equations 77 (1989) 254-286.

[12] J. Prüss, On the spectrum of $C_{0}$-semigroups, Trans. Amer. Math. Soc. 284 (2) (1984) 847-857.

[13] R.K. Yedavalli, Perturbation bounds for robust stability in linear state space models. Internat. J. Control 42 (1985) $1507-1517$. 